\title{
Harmonic oscillator Brownian motion: Langevin approach revisited
}

\author{
O. Contreras-Vergara, N. Lucero-Azuara, and N. Sánchez-Sala \\ Departamento de Física, Escuela Superior de Física y Matemáticas, \\ Instituto Politécnico Nacional, Edif. 9 UP Zacatenco, 07738, CDMX, México. \\ e-mail: nsanchezs@ipn.mx \\ J. I. Jiménez-Aquino \\ Departamento de Física, Universidad Autónoma, Metropolitana-Iztapalapa, \\ 09340, CDMX, México. \\ e-mail: ines@xanum.uam.mx
}

Received 29 June 2020; accepted 5 September 2020

\begin{abstract}
The original strategy applied by Langevin to the Brownian movement problem is used to solve the case of a free particle under a harmonic potential. Such a straightforward strategy consists of separating the noise term in the Langevin equation to solve a deterministic equation associated with the Mean Square Displacement. In this work, we use the Langevin's original strategy to calculate the statistical properties of the harmonic oscillator Brownian Motion, in the damped and periodic cases. It is shown that, in the long time limit, Langevin's original method is consistent with the exact theoretical solutions reported by Chandrasekhar and Lemons, these latter obtained using the statistical properties of a Gaussian white noise. Also, unexpected results are presented when the method is applied to a free particle case. Our results are compared with the exact theoretical solutions, as well as with the numerical simulations.
\end{abstract}

Keywords: Langevin equation; Brownian motion.

PACS: $05.10 . \mathrm{Gg}$; 05.40.Jc

DOI: https://doi.org/10.31349/RevMexFisE.18.97

\section{Introducction}

In 1908 the physicist P. Langevin [1] presented a straightforward method than Einstein's theory [2] to solve the problem of Brownian motion [3,4]. In his original paper, Langevin proposed a solution based on Newton's 2nd Law, $m a=F$, $F$ being the net force acting on the particle; in this case, the friction force (Stokes law) plus a time-dependent fluctuating force (the noise term). This equation bears his name, and it is also called a stochastic differential equation. Langevin's purposes was to obtain through the solution of his equation the same mean square displacement (MSD) previously reported by Einstein, that is $\left\langle x^{2}(t)\right\rangle=2 D t$, being $D=$ $k_{B} T / 6 \pi \eta r$ Einstein's diffusion coefficient, $\eta$ the viscosity of the fluid and $r$ the radius of the particle. The strategy used by Langevin to solve the problem was ingenious and simple; it consists of eliminating the fluctuating force and then obtaining a deterministic equation for the MSD. He also had to introduce two important hypotheses: one is the independence between both the fluctuating force and the mean trajectory of the particle, and the other, the energy equipartition theorem in thermal equilibrium. Finally, for times larger than the relaxation time, Langevin found Einstein's result for the MSD.

Nowadays, the Langevin equation or a Langevin-type equation is widely used in diverse systems in different fields such as physics, chemistry, biology, etc. After the publication of Langevin's work, other outstanding contributions to the theory of Brownian motion appeared in a collection of papers on noise and stochastic processes [4]. Practically all the academic and research works related to the study of Brow- nian motion has been developed using the standard method in the context of the Langevin equation or the Fokker-Planck equation taking into account the Gaussian statistical properties of noise [4-26]. There exists a variety of methods for solving the Langevin and Fokker-Planck equations, for instance, Laplace transforms, Fourier transforms, Green's function method, the variation of parameters, etc. Here, Chandrasekhar's pioneering contribution must be highlighted because, in his paper, the problem of harmonic oscillator Brownian motion (HOBM) was explicitly solved by the usual methods of stochastic processes [5]. Lemons [6] also studied the problem using the Ito-Type stochastic differential equation upon the solution of this equation was in terms of differentials, he was able to calculate all the statistical properties of a Brownian Harmonic oscillator. Lemons' results are the same as those reported by Chandrasekhar. As well, an important amount of research and academic works related to HOBM has been reported in the literature [17-23]. Other interesting and recent publications of experimental academic works have been reported; for super paramagnetic colloids [24] and a repetition of Perrin's experiments [26].

Our purpose, in the present academic contribution, is to use Langevin's original strategy to study the statistical properties of a HOBM in the damped and periodic cases. In this case, all the solutions are approximated but consistent with the exact $[5,6]$, and numerical simulations result in the long time limit. It is shown that, in the short time limit, Langevin's methodology mostly it doesn't work. To a better understanding of Langevin's strategy, we first study the free particle problem, for which we have found interesting results. In this 
case, the MSD becomes the same as the exact result only when the initial velocity is assumed to satisfy the Maxwell distribution function; otherwise, the MSD disagree. For the Ornstein-Uhlenbeck (OU) process, Langevin's original strategy reproduces the same result as the exact method, which is a surprising result. Langevin's method can be useful for students of Brownian motion graduate courses, since it is simpler to apply and gives results that are close to exact, which are shown throughout the work.

Our work is structured as follows: As pedagogical support we study in Sec. 2 Langevin's original approach to calculate the MSD and MSV for a free Brownian particle. Section 3 focuses on Langevin's strategy to solve the statistical properties associated with a harmonic oscillator in the damped and periodic cases. Our solutions for HOBM are compared with Chandrasekhar's and Lemons' results as well as numerical simulations. In Sec. 4, the conclusions are enunciated, and we add an Appendix at the end of our work.

\section{Free particle Brownian motion}

\subsection{Mean square displacement}

In this section, we review the Langevin original strategy to calculate the MSD for a free Brownian particle of mass $m$, embedded in a thermal bath of temperature $T$. In the case, the Langevin equation can be written as [1]

$$
m \frac{d^{2} x}{d t^{2}}=-\alpha \frac{d x}{d t}+\xi(t)
$$

where $\alpha$ is the friction coefficient, and $\xi(t)$ is the fluctuating force. The first step given by Langevin was to multiply Eq. (1) by $x$ to obtain

$$
m x \frac{d^{2} x}{d t^{2}}=-\alpha x \frac{d x}{d t}+x \xi(t) .
$$

Then, he transformed this equation into

$$
\frac{1}{2} \frac{d^{2} x^{2}}{d t^{2}}-v^{2}=-\frac{\beta}{2} \frac{d x^{2}}{d t}+\frac{1}{m} x \xi(t),
$$

where $\beta=\alpha / m$. Next, Langevin took the average ensemble for several identical particles to get

$$
\frac{1}{2} \frac{d^{2}\left\langle x^{2}\right\rangle}{d t^{2}}=-\frac{\beta}{2} \frac{d\left\langle x^{2}\right\rangle}{d t}+\left\langle v^{2}\right\rangle+\frac{1}{m}\langle x \xi(t)\rangle .
$$

To continue, two crucial hypotheses were considered, namely: (i) Due to the irregularity of random force $\xi(t)$, he considered that the average value $\langle\xi(t) x\rangle=0$. (ii) The validity of the energy equipartition theorem, that is, in thermal equilibrium, the average kinetic energy satisfies $(1 / 2) m\left\langle v^{2}\right\rangle=(1 / 2) k_{B} T$, and under these conditions, Eq. (4) is rewritten as

$$
\frac{d^{2}\left\langle x^{2}\right\rangle}{d t^{2}}=-\beta \frac{d\left\langle x^{2}\right\rangle}{d t}+2 \frac{k_{B} T}{m} .
$$

For a first integration, the solution reads

$$
\frac{d\left\langle x^{2}\right\rangle}{d t}=\frac{2 k_{B} T}{m \beta}-\frac{2 k_{B} T}{m \beta} e^{-\beta t},
$$

where $d\left\langle x^{2}(t)\right\rangle /\left.d t\right|_{0}=0$. For a second integration, we get

$$
\left\langle x^{2}(t)\right\rangle_{L}-\left\langle x^{2}(0)\right\rangle=\frac{2 k_{B} T}{\beta m} t-\frac{2 k_{B} T}{m \beta^{2}}\left(1-e^{-\beta t}\right) .
$$

the Langevin result.

On the other hand, using the statistical properties of Gaussian white noise, it can be shown that the exact result for the MSD reads

$$
\begin{aligned}
\left\langle x^{2}(t)\right\rangle_{E}-\left\langle x^{2}(0)\right\rangle & =\left(v_{0}^{2}-\frac{q}{2 \beta}\right) \frac{\left(1-e^{. \beta t}\right)^{2}}{\beta^{2}} \\
& +\frac{q}{\beta^{2}} t-\frac{q}{\beta^{3}}\left(1-e^{-\beta t}\right),
\end{aligned}
$$

where $q=2 \beta k_{B} T / m$. It is well known that, Langevin Eq. (1) for the velocity is stationary, and it satisfies the Maxwell distribution function with zero mean value and variance $\sigma_{v}^{2}=\left\langle v^{2}\right\rangle_{s t}=k_{B} T / m$. In fact, this is one of the hypotheses used by Langevin in Eq. (4) to solve the problem. Due to the stationary character of the velocity also $\left\langle v_{0}^{2}\right\rangle=k_{B} T / m=q / 2 \beta$ and therefore, the first term in the right-hand side of Eq. (8) vanishes. In this case, the exact solution (8) for the MSD reduces to Eq. (7), obtained from Langevin's methodology. It is clear that, if the initial velocity is fixed, then the exact solution does not coincide with the Langevin result. That is, if the velocity is initially distributed with a Maxwell distribution, then the exact solution $(8)$ is equal to the one obtained by Langevin's strategy.

In the short time limit, Eq. (7) reduces to $\left\langle x^{2}(t)\right\rangle=$ $\left(k_{B} T / m\right) t^{2}$, which corresponds to the ballistic regime, whereas in the large time limit, it becomes

$$
\left\langle\left\langle x^{2}(t)\right\rangle-\left\langle x^{2}(0)\right\rangle=2 D t\right.
$$

the same Einstein's result. In Langevin's original paper, he did not show the analysis for short times because his main goal was to recover the Einstein result, and it is obtained in the long time limit.

In Fig. 1, we compare both MSD (7), (8) with the numerical simulation [27] of Eq. (1), when $v_{0}^{2} \neq(q / 2 \beta)$. In this case, the result obtained by Langevin's method (red) separates from the exact (black) and numerical simulation (black circles) in the region of short times, but all of them coincide in the long time limit.

\subsection{Mean Square Velocity (MSV)}

In this section, we now investigate if the Langevin original strategy works for a free particle MSV, corresponding to the Ornstein-Uhlenbeck (OU) process. For this case, we write

$$
m \frac{d v}{d t}=-\alpha v+\xi(t) .
$$




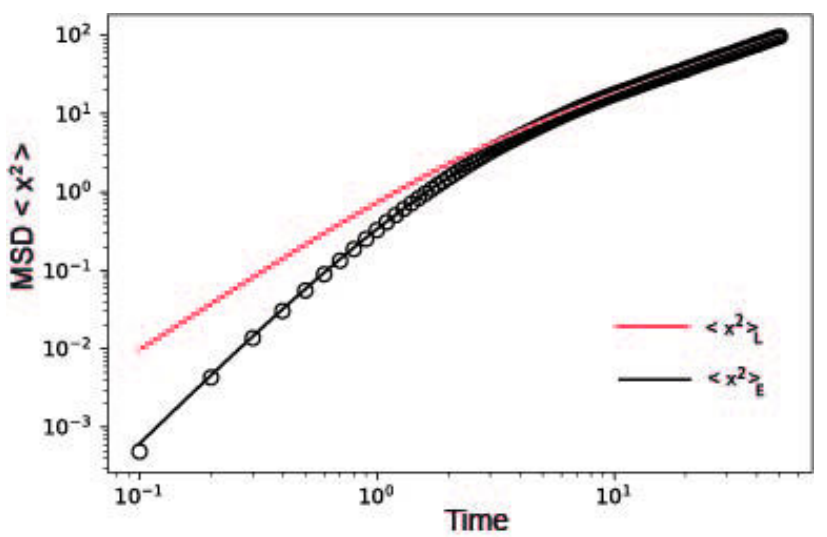

FIGURE 1. Comparison between MSD (7) (red), (8) (black), and simulation results (circles), with $v_{0}=0, k_{B} T=m=\beta=1.0$.

Multiplying this equation by $v$ and taking the ensemble average, we get

$$
\frac{d\left\langle v^{2}\right\rangle}{d t}=-2 \beta\left\langle v^{2}\right\rangle+\frac{2}{m}\langle v \xi(t)\rangle .
$$

Next, to separate the noise term in this equation, we follow the Langevin idea. In this case, the correlation function $\langle v \xi(t)\rangle$ must not be equal to zero, as one could believe, instead, we assume that it is constant, $\langle v \xi(t)\rangle=C_{0}$. This assumption allows to easily obtain the solution for the Mean Square Velocity (MSV), which is given by the solution of

$$
\frac{d\left\langle v^{2}\right\rangle}{d t}=-2 \beta\left\langle v^{2}\right\rangle+\frac{2 C_{0}}{m} .
$$

Its solution reads

$$
\left\langle v^{2}(t)\right\rangle_{L}=\left\langle v_{0}^{2}\right\rangle e^{-2 \beta t}+\frac{C_{0}}{\alpha}\left[1-e^{-2 \beta t}\right] .
$$

As can be seen, $C_{0}$ can be determined by imposing the validity of the energy equipartition theorem at thermal equilibrium. Hence, in the long time limit

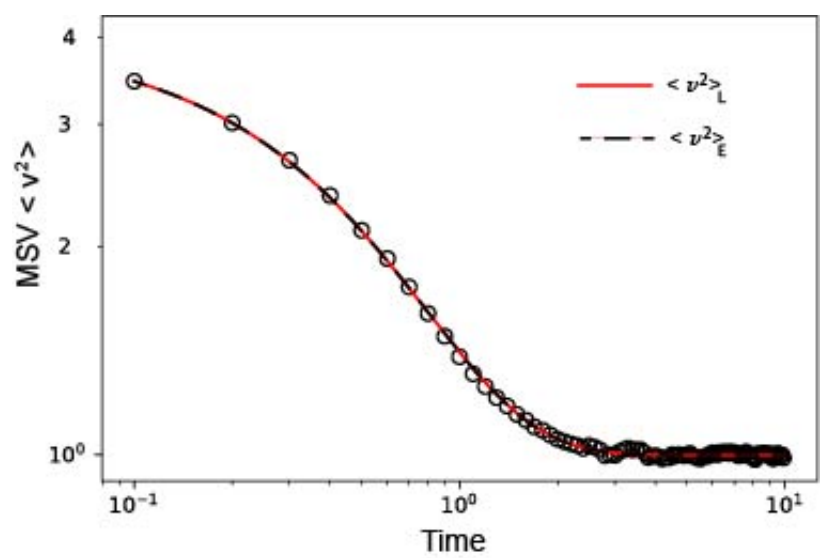

FIGURE 2. Comparison of the MSV (15) with the exact solution and numerical simulation. All normalized by the factor $k_{B} T / m$, $v_{0}=2.0$.

$$
\left\langle v^{2}\right\rangle_{s t}=\frac{C_{0}}{\alpha}=\frac{k_{B} T}{m}, \quad C_{0}=\frac{\alpha k_{B} T}{m} .
$$

Therefore, the MSV becomes

$$
\left\langle v^{2}(t)\right\rangle_{L}=\left\langle v_{0}^{2}\right\rangle e^{-2 \beta t}+\frac{k_{B} T}{m}\left[1-e^{-2 \beta t}\right] .
$$

If we want to obtain the exact solution for MSV, $\left\langle v^{2}(t)\right\rangle_{E}$, we have to use the statistical properties of a Gaussian white noise, curiously, in this case, $\left\langle v^{2}(t)\right\rangle_{E}=\left\langle v^{2}(t)\right\rangle_{L}$. Surprisingly, Langevin's strategy not only works to obtain the free particle MSV but also becomes the same as the exact solution. In Fig. 2, we show the MSV, Eq. (15) (red) with both the exact solution (black) and numerical simulation results (black circles).

\section{Harmonic oscillator Brownian motion}

\subsection{Mean square displacement}

\subsubsection{Dammped case $(\beta>2 \omega)$}

We proceed to apply Langevin's original strategy to study the problem of the harmonic oscillator Brownian motion. Here, we calculate the variance and the MSD for the above free particle bounded by a harmonic potential for which the Langevin equation can be written as

$$
\frac{d^{2} x}{d t^{2}}+\beta \frac{d x}{d t}+\omega^{2} x=\frac{1}{m} \xi(t),
$$

where $\omega^{2}=k / m$ is the harmonic oscillator's characteristic frequency. To obtain the deterministic equation associated with the variance $\sigma_{x}^{2}(t)=\left\langle x^{2}(t)\right\rangle-\langle x(t)\rangle^{2}$, we first multiply Eq. (16) by $x$ and thus

$$
x \frac{d^{2} x}{d t^{2}}+\beta x \frac{d x}{d t}+\omega^{2} x^{2}=\frac{1}{m} x \xi(t) .
$$

However, we can see that

$$
\frac{d^{2} x^{2}}{d t^{2}}=2 x \frac{d^{2} x}{d t^{2}}+2\left(\frac{d x}{d t}\right)^{2}, \quad \frac{d x^{2}}{d t}=2 x \frac{d x}{d t},
$$

and so Eq. (17) transforms into

$$
\frac{d^{2} x^{2}}{d t^{2}}-2 v^{2}+\beta \frac{d x^{2}}{d t}+2 \omega^{2} x^{2}=\frac{2}{m} x \xi(t) .
$$

Taking the ensemble average and assuming also that $\langle x \xi(t)\rangle=0$, we have

$$
\frac{d^{2}\left\langle x^{2}\right\rangle}{d t^{2}}+\beta \frac{d\left\langle x^{2}\right\rangle}{d t}+2 \omega^{2}\left\langle x^{2}\right\rangle=2\left\langle v^{2}\right\rangle .
$$

On the other hand, we now take the ensemble average of Eq. (16) to obtain

$$
\frac{d^{2}\langle x\rangle}{d t^{2}}+\beta \frac{d\langle x\rangle}{d t}+\omega^{2}\langle x\rangle=0
$$


where $\langle\xi(t)\rangle=0$, because Eq. (21) has to coincide with the harmonic oscillator deterministic equation. We now multiply Eq. (21) by $\langle x\rangle$ to get

$$
\langle x\rangle \frac{d^{2}\langle x\rangle}{d t^{2}}+\beta\langle x\rangle \frac{d\langle x\rangle}{d t}+\omega^{2}\langle x\rangle^{2}=0
$$

but also

$$
\begin{aligned}
\frac{d^{2}\langle x\rangle^{2}}{d t^{2}} & =2\langle x\rangle \frac{d^{2}\langle x\rangle}{d t^{2}}+2\left(\frac{d\langle x\rangle}{d t}\right)^{2}=2\langle x\rangle \frac{d^{2}\langle x\rangle}{d t^{2}}+2\langle v\rangle^{2} \\
\frac{d\langle x\rangle^{2}}{d t} & =2\langle x\rangle \frac{d\langle x\rangle}{d t} .
\end{aligned}
$$

In this case, Eq. (22) becomes

$$
\frac{d^{2}\langle x\rangle^{2}}{d t^{2}}+\beta \frac{d\langle x\rangle^{2}}{d t}+2 \omega^{2}\langle x\rangle^{2}=2\langle v\rangle^{2} .
$$

From Eqs. (20) and (24), we clearly see that the deterministic equation for the variance reads

$$
\frac{d^{2} \sigma_{x}^{2}}{d t^{2}}+\beta \frac{d \sigma_{x}^{2}}{d t}+2 \omega^{2} \sigma_{x}^{2}=2\left(\left\langle v^{2}\right\rangle-\langle v\rangle^{2}\right) .
$$

We take into account the second hypothesis proposed by Langevin related to the energy equipartition theorem, which is obtained from the Maxwell equilibrium distribution. For such distribution the mean value is $\langle v\rangle=0$ and $(1 / 2) m\left\langle v^{2}\right\rangle=(1 / 2) k_{B} T$, and therefore

$$
\frac{d^{2} \sigma_{x}^{2}}{d t^{2}}+\beta \frac{d \sigma_{x}^{2}}{d t}+2 \omega^{2} \sigma_{x}^{2}=2 \frac{k_{B} T}{m}
$$

which is the deterministic equation associated with the variance. Its solution can be calculated using the undetermined

coefficients method. First, we solve the homogeneous equation given by

$$
\frac{d^{2} \sigma_{x h}^{2}}{d t^{2}}+\beta \frac{d \sigma_{x h}^{2}}{d t}+2 \omega^{2} \sigma_{x h}^{2}=0
$$

and its solution in the damped case $(\beta>\sqrt{8} \omega)$ reads

$$
\begin{aligned}
\sigma_{x h}^{2} & =C_{1} e^{\left(-\frac{\beta}{2}-\frac{1}{2} \sqrt{\beta^{2}-8 \omega^{2}}\right) t} \\
& +C_{2} e^{\left(-\frac{\beta}{2}+\frac{1}{2} \sqrt{\beta^{2}-8 \omega^{2}}\right) t} .
\end{aligned}
$$

The particular solution is a constant $\sigma_{x p}^{2}=A$, which is substituted into Eq. (27) to get

$$
\sigma_{x p}^{2}=\frac{k_{B} T}{m \omega^{2}}
$$

So the solution of Eq. (26) is given by $\sigma_{x}^{2}=\sigma_{x h}^{2}+\sigma_{x p}^{2}$, and thus

$$
\begin{aligned}
\sigma_{x}^{2}(t) & =C_{1} e^{\left(-\frac{\beta}{2}-\frac{1}{2} \sqrt{\beta^{2}-8 \omega^{2}}\right) t} \\
& +C_{2} e^{\left(-\frac{\beta}{2}+\frac{1}{2} \sqrt{\beta^{2}-8 \omega^{2}}\right) t}+\frac{k_{B} T}{m \omega^{2}} .
\end{aligned}
$$

The constants $C_{1}$ and $C_{2}$ can be obtained from the initial conditions $\sigma_{x}^{2}(0)=0$ and $d \sigma_{x}^{2}(0) / d t=0$, so that

$$
\begin{aligned}
& C_{1}=\frac{k_{B} T \beta}{2 m \omega^{2} \sqrt{\beta^{2}-8 \omega^{2}}}-\frac{k_{B} T}{2 m \omega^{2}}, \\
& C_{2}=-\frac{k_{B} T \beta}{2 m \omega^{2} \sqrt{\beta^{2}-8 \omega^{2}}}-\frac{k_{B} T}{2 m \omega^{2}} .
\end{aligned}
$$

Hence, the solution of Eq. (26) can be written as

$$
\sigma_{x_{O}}^{2}(t)=\frac{k_{B} T}{m \omega^{2}}\left\{1-e^{-\frac{1}{2} \beta t}\left(2 \sinh ^{2} \frac{1}{4} \bar{\beta}_{1} t+\frac{\beta}{\bar{\beta}_{1}} \sinh \frac{1}{2} \bar{\beta}_{1} t+1\right)\right\},
$$

where, $\bar{\beta}_{1}=\sqrt{\beta^{2}-8 \omega^{2}}$. In this case, The MSD

$$
\left\langle x^{2}(t)\right\rangle_{O}=\langle x(t)\rangle^{2}+\frac{k_{B} T}{m \omega^{2}}\left\{1-e^{-\frac{1}{2} \beta t}\left(2 \sinh ^{2} \frac{1}{4} \bar{\beta}_{1} t+\frac{\beta}{\bar{\beta}_{1}} \sinh \frac{1}{2} \bar{\beta}_{1} t+1\right)\right\},
$$

and the solution for the average $\langle x(t)\rangle$ comes from the explicit solution of Eq. (21), that is

$$
\langle x(t)\rangle=x_{0} e^{-\frac{1}{2} \beta t}\left(\cosh \frac{1}{2} \beta_{1} t+\frac{\beta}{\beta_{1}} \sinh \frac{1}{2} \beta_{1} t\right)+\frac{2 v_{0}}{\beta_{1}} e^{-\frac{1}{2} \beta t} \sinh \frac{1}{2} \beta_{1} t,
$$

being $\beta_{1}=\sqrt{\beta^{2}-4 \omega^{2}}$, and $x_{0}, v_{0}$ the initial conditions for position and velocity, respectively. Equation (33) is similar to those obtained by Chandrasekhar and Lemons, being Chandrasekhar's result the following expression: (see Eq. (214) in Ref. [5])

$$
\left\langle x^{2}(t)\right\rangle_{C}=\langle x(t)\rangle^{2}+\frac{k_{B} T}{m \omega^{2}}\left\{1-e^{-\beta t}\left(2 \frac{\beta^{2}}{\beta_{1}^{2}} \sinh ^{2} \frac{1}{2} \beta_{1} t+\frac{\beta}{\beta_{1}} \sinh \beta_{1} t+1\right)\right\},
$$

where $\langle x(t)\rangle$ is the same as Eq. (34). Lemons' result is the same as Eq. (35), and it is given by Eq. (A.6) in Appendix A. 


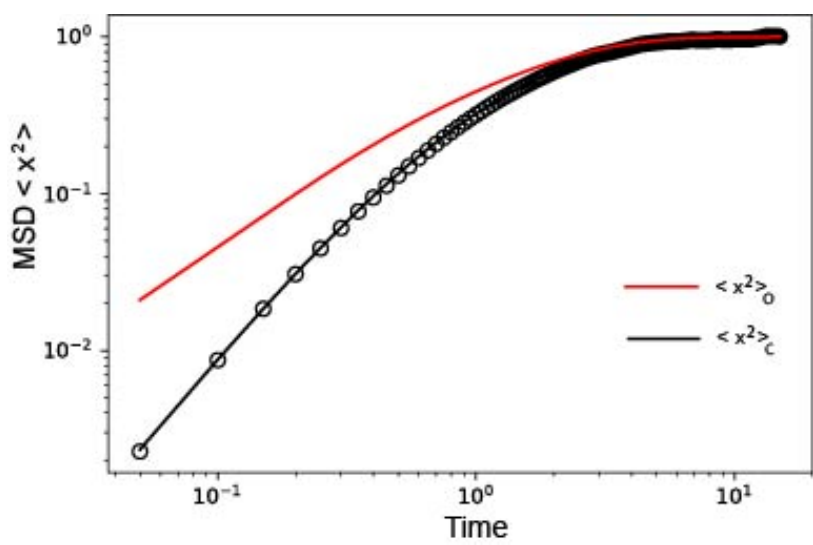

FIGURE 3. Comparison between MSDs (33) (red), (35) (black) and numerical simulation (circles). All normalized by the factor $k_{B} T / m \omega^{2}$ and $x_{0}=0$ and $v_{0}=1.0$.

As we can see, the structure of Eq. (33) is a little bit different from Eq. (35) because the parameter $\bar{\beta}_{1} \neq \beta_{1}$, and the ratio $\beta^{2} / \beta_{1}^{2}$ that appears in Eq. (35) does no in Eq. (33). The comparison between both MSD is shown in Fig. 3, where we can observe certain deviations in the transient region. However, as time increases, both MSD reach the same stationary state value. In Fig. 3, the simulation results (circles) for the MSD coincide with Chandrasekhar's and Lemons' results (black curve), and they match with our result (red curve) (33) as the time gets large.

It is clear that, for $\beta t \gg 1$, the mean value $\langle x\rangle \rightarrow 0$ and the MSDs in both treatments become

$$
\left\langle x^{2}\right\rangle_{s t}=\frac{k_{B} T}{m \omega^{2}} .
$$

Thus, Langevin's original methodology is consistent in describing the MSD for harmonic oscillator Brownian motion in the large time limit. We clarify that, the deviations from the exact solutions, as shown in the transient region of Fig. 3, come from having initially eliminated the noise term in the Langevin equation, causing a lack of information for a complete solution to the problem.

\subsubsection{Periodic case $(\beta<2 \omega)$}

For the harmonic oscillator in the periodic case, after some algebra, we can show that the solution of Eq. (26) can be written as

$$
\begin{aligned}
& \sigma_{x_{O}}^{2}(t)=\frac{k_{B} T}{m \omega^{2}}\left\{1-e^{-\frac{1}{2} \beta t}\right. \\
& \left.\times\left(-2 \sin ^{2} \frac{\sqrt{2}}{2} \bar{\omega}_{1} t+\frac{\beta}{\sqrt{8} \bar{\omega}_{1}} \sin \sqrt{2} \bar{\omega}_{1} t+1\right)\right\},
\end{aligned}
$$

where $\bar{\omega}_{1}=\sqrt{\omega^{2}-\left(\beta^{2} / 8\right)}$, and then the MSD becomes

$$
\begin{aligned}
& \left\langle x^{2}(t)\right\rangle_{O}=\langle x(t)\rangle^{2}+\frac{k_{B} T}{m \omega^{2}}\left\{1-e^{-\frac{\beta}{2} t}\right. \\
& \left.\times\left(-2 \sin ^{2} \frac{\sqrt{2}}{2} \bar{\omega}_{1} t+\frac{\beta}{\sqrt{8} \bar{\omega}_{1}} \sin \sqrt{2} \bar{\omega}_{1} t+1\right)\right\},
\end{aligned}
$$

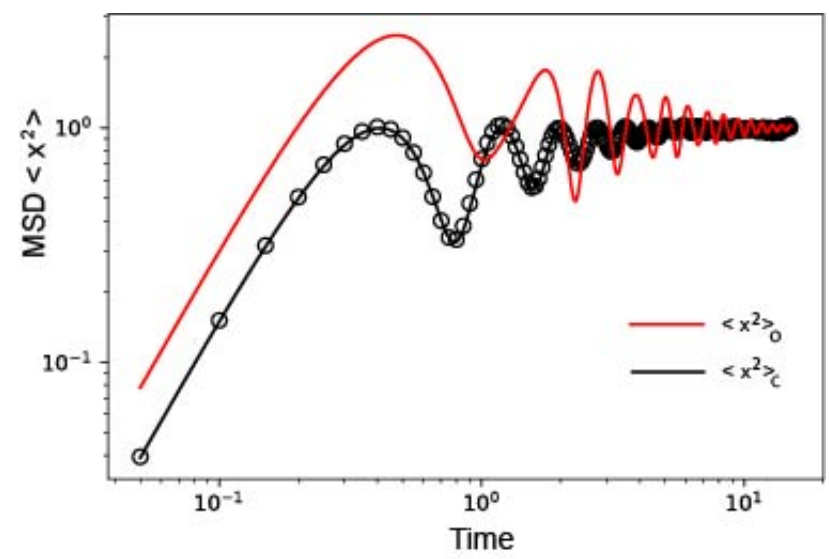

FIGURE 4. Comparison between MSD (38) (red), (40) (black) and numerical simulation (circles). All normalized by the factor $k_{B} T / m \omega^{2}$, with $x_{0}=0$ and $v_{0}=1.0$.

being the mean value

$$
\begin{aligned}
\langle x(t)\rangle & =x_{0} e^{-\frac{1}{2} \beta t}\left(\cos \omega_{1} t+\frac{\beta}{2 \omega_{1}} \sin \omega_{1} t\right) \\
& +\frac{v_{0}}{\omega_{1}} e^{-\frac{1}{2} \beta t} \sin \omega_{1} t
\end{aligned}
$$

with $\omega_{1}=\sqrt{\omega^{2}-\left(\beta^{2} / 4\right)}$. On the other side, the MSD proposed by Chandrasekhar in the periodic case reads

$$
\begin{aligned}
\left\langle x^{2}\right\rangle_{C} & =\langle x(t)\rangle^{2}+\frac{k_{B} T}{m \omega^{2}}\left\{1-e^{-\beta t}\right. \\
& \left.\times\left(\frac{\beta^{2}}{2 \omega_{1}^{2}} \sin ^{2} \omega_{1} t+\frac{\beta}{2 \omega_{1}} \sin 2 \omega_{1} t+1\right)\right\},
\end{aligned}
$$

where the mean value $\langle x(t)\rangle$ is the same as Eq. (39).

The MSD (40) is the same result (A.3) reported by Lemons (see Appendix A). Again our theoretical result (38) has a similar structure as the one given by Eq. (40). This fact is shown in Fig. 4, where we can see deviations from the exact solution in the transient region; however, as time increases, both results tend to the stationary state value $k_{B} T / m \omega^{2}$, as expected. This confirms the consistency of Langevin's original approach in the large time limit. Similarly as we have commented in the damped case, the deviations in the transient region come from the separation of the noise term in the Langevin equation.

\subsection{Mean square velocity (MSV)}

Let us now apply the Langevin approach to the case of HOBM in the velocity space. In this case, due to the presence of the harmonic force, the cross correlation function plays a role. We start with the Langevin equation

$$
\frac{d v}{d t}=-\beta v-\omega^{2} x+\frac{1}{m} \xi(t) .
$$


Again we multiply by $v$ and take the ensemble average to get

$$
\frac{d\left\langle v^{2}\right\rangle}{d t}=-2 \beta\left\langle v^{2}\right\rangle-2 \omega^{2}\langle x v\rangle+\frac{2}{m}\langle v \xi(t)\rangle .
$$

Considering the hypothesis that $\langle v \xi(t)\rangle=C_{1}=c t e$, and thus

$$
\frac{d\left\langle v^{2}\right\rangle}{d t}=-2 \beta\left\langle v^{2}\right\rangle-2 \omega^{2}\langle x v\rangle+\frac{2 C_{1}}{m} .
$$

To obtain the velocity variance $\sigma_{v}^{2}=\left\langle v^{2}\right\rangle-\langle v\rangle^{2}$, we need to do the following: we take the ensemble average of Eq. (41) and multiply by $\langle v\rangle$ in such a way that

$$
\frac{d\langle v\rangle^{2}}{d t}=-2 \beta\langle v\rangle^{2}-2 \omega^{2}\langle x\rangle\langle v\rangle \text {. }
$$

From Eqs. (43) and (44) we obtain the differential equation for the velocity variance, given by

$$
\frac{d \sigma_{v}^{2}}{d t}=-2 \beta \sigma_{v}^{2}-2 \omega^{2} \sigma_{x v}^{2}+\frac{2 C_{1}}{m},
$$

where $\sigma_{x v}^{2}=\langle x v\rangle-\langle x\rangle\langle v\rangle$ is the $x$ and $v$ covariance. On the other hand, from the definition $v=d x / d t$, it is easy to see that

$$
\sigma_{x v}^{2}=\frac{1}{2} \frac{d \sigma_{x}^{2}}{d t}
$$

Hence, the velocity variance can be calculated once the covariance is obtained from Eq. (46).

\subsection{1. $\quad$ Damped case $(\beta>2 \omega)$}

In this case, we use the result given by Eq. (32), and according to Eq. (46), we can show that

$$
\sigma_{x v}^{2}=\frac{2 k_{B} T}{m \bar{\beta}_{1}} e^{-\frac{1}{2} \beta t} \sinh \left(\frac{1}{2} \bar{\beta}_{1} t\right) .
$$

Upon substitution of Eq. (47) into Eq. (45), we now get

$$
\begin{aligned}
\frac{d \sigma_{v}^{2}}{d t} & =-2 \beta \sigma_{v}^{2}-\frac{4 k_{B} T \omega^{2}}{m \bar{\beta}_{1}} e^{-\frac{1}{2} \beta t} \\
& \times \sinh \left(\frac{1}{2} \bar{\beta}_{1} t\right)+\frac{2 C_{1}}{m} .
\end{aligned}
$$

The solution of this equation becomes

$$
\begin{aligned}
\sigma_{v_{O}}^{2}(x) & =\left(\sigma_{0}^{2}-\frac{k_{B} T \omega^{2}}{m\left(\beta^{2}+\omega^{2}\right)}\right) e^{-2 \beta t}+\frac{k_{B} T \omega^{2}}{m\left(\beta^{2}+\omega^{2}\right)} \\
& \times e^{-\frac{1}{2} \beta t}\left[2 \sinh ^{2}\left(\frac{1}{4} \bar{\beta}_{1} t\right)-\frac{3 \beta}{\bar{\beta}_{1}} \sinh \left(\frac{1}{2} \bar{\beta}_{1} t\right)+1\right] \\
& +\frac{C_{1}}{\beta m}\left(1-e^{-\frac{1}{2} \beta t}\right)
\end{aligned}
$$

so that

$$
\begin{aligned}
\left\langle v^{2}(t)\right\rangle_{O} & =\langle v(t)\rangle^{2}+\left(\sigma_{0}^{2}-\frac{k_{B} T \omega^{2}}{m\left(\beta^{2}+\omega^{2}\right)}\right) e^{-2 \beta t} \\
+ & \frac{k_{B} T \omega^{2}}{m\left(\beta^{2}+\omega^{2}\right)} e^{-\frac{1}{2} \beta t}\left[2 \sinh ^{2}\left(\frac{1}{4} \bar{\beta}_{1} t\right)\right. \\
& \left.-\frac{3 \beta}{\bar{\beta}_{1}} \sinh \left(\frac{1}{2} \bar{\beta}_{1} t\right)+1\right]+\frac{C_{1}}{\beta m}\left(1-e^{-\frac{1}{2} \beta t}\right)
\end{aligned}
$$

being the average value $\langle v(t)\rangle$ the deterministic solution of Eq. (41), and given by

$$
\begin{aligned}
\langle v(t)\rangle & =v_{0} e^{-\frac{1}{2} \beta t}\left[\cosh \left(\frac{1}{2} \beta_{1} t\right)-\frac{\beta}{\beta_{1}} \sinh \left(\frac{1}{2} \beta_{1} t\right)\right] \\
& -\frac{2 x_{0} \omega^{2}}{\beta_{1}} e^{-\frac{1}{2} \beta t} \sinh \left(\frac{1}{2} \beta_{1} t\right)
\end{aligned}
$$

Again the constant $C_{1}$ can be determined by the energy equipartition theorem at equilibrium. As time increases it is clear that that $\left\langle v^{2}(t)\right\rangle_{O} \rightarrow\left(C_{1} / \alpha\right)$, and thus $C_{1}=\alpha k_{B} T / m$, which is the same constant $C_{0}$ obtained before.

The exact solution reported by Chandrasekhar [5] in the damped case reads

$$
\begin{aligned}
\left\langle v^{2}(t)\right\rangle_{C} & =\langle v(t)\rangle^{2}+\frac{k_{B} T}{m}\left\{1-e^{-\beta t}\left[2 \frac{\beta^{2}}{\beta_{1}^{2}} \sinh ^{2}\left(\frac{1}{2} \beta_{1} t\right)\right.\right. \\
& \left.\left.-\frac{\beta}{\beta_{1}} \sinh \left(\beta_{1} t\right)+1\right]\right\},
\end{aligned}
$$

where $\langle v(t)\rangle$ is the same as Eq. (51). In the long time limit, the exact solution becomes $\left\langle v(t)^{2}\right\rangle_{C}=k_{B} T / m$, also consistent with the energy equipartition theorem. The structure of the solution (50) is different from the exact solution (52); however, in the long time limit both coincide, as expected. The comparison of the MSV (50) with the exact and numerical simulation results is shown in Fig. 5, where the coincidence of the three results is clearly shown in the long time limit. Discrepancies are observed at short and intermediate times.

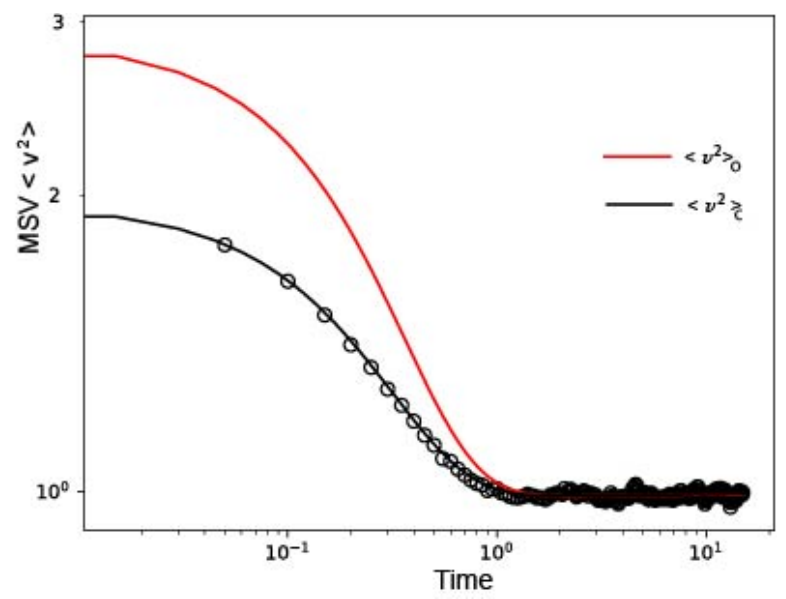

FIGURE 5. Comparison of MSV (50) (red) with both, exact (black) and numerical simulation (circles) results, for values $v_{0}=1.4 \mathrm{y}$ $x_{0}=0.5$. 


\subsubsection{Periodic case $(\beta<2 \omega)$}

For this case, we now use the result given by Eq. (37) and, after some algebra, we can show that

$$
\begin{aligned}
\sigma_{v_{O}}^{2} & =\left(\sigma_{0}^{2}-\frac{k_{B} T \omega^{2}}{m\left(\omega^{2}+\beta^{2}\right)}\right) e^{-2 \beta t}+\frac{k_{B} T \omega^{2}}{m\left(\omega^{2}+\beta^{2}\right)} e^{-\frac{1}{2} \beta t} \\
& \times\left[\cos \left(\sqrt{2} \bar{\omega}_{1} t\right)-\frac{3 \beta}{\sqrt{8} \bar{\omega}_{1}} \sin \left(\sqrt{2} \bar{\omega}_{1} t\right)\right] \\
& +\frac{C_{1}}{\beta m}\left(1-e^{-2 \beta t}\right)
\end{aligned}
$$

and thus

$$
\begin{aligned}
\left\langle v^{2}(t)\right\rangle_{O} & =\langle v(t)\rangle^{2}+\left(\sigma_{0}^{2}-\frac{k_{B} T \omega^{2}}{m\left(\omega^{2}+\beta^{2}\right)}\right) e^{-2 \beta t} \\
+ & \frac{k_{B} T \omega^{2}}{m\left(\omega^{2}+\beta^{2}\right)} e^{-\frac{1}{2} \beta t}\left[\cos \left(\sqrt{2} \bar{\omega}_{1} t\right)\right. \\
- & \left.\frac{3 \beta}{\sqrt{8} \bar{\omega}_{1}} \sin \left(\sqrt{2} \bar{\omega}_{1} t\right)\right]+\frac{C_{1}}{\beta m}\left(1-e^{-2 \beta t}\right) .
\end{aligned}
$$

where the average value $\langle v(t)\rangle$ is

$$
\begin{aligned}
\langle v(t)\rangle & =v_{0} e^{-\frac{1}{2} \beta t}\left[\cos \left(\omega_{1} t\right)-\frac{\beta}{2 \omega_{1}} \sin \left(\omega_{1} t\right)\right] \\
& -\frac{x_{0} \omega^{2}}{\omega_{1}} e^{-\frac{1}{2} \beta t} \sin \left(\omega_{1} t\right) .
\end{aligned}
$$

Again, for long times we show that $C_{1}=\alpha K_{B} T / m$, due to the energy equipartition theorem. The exact result reported by Chandrasekhar for the periodic case is

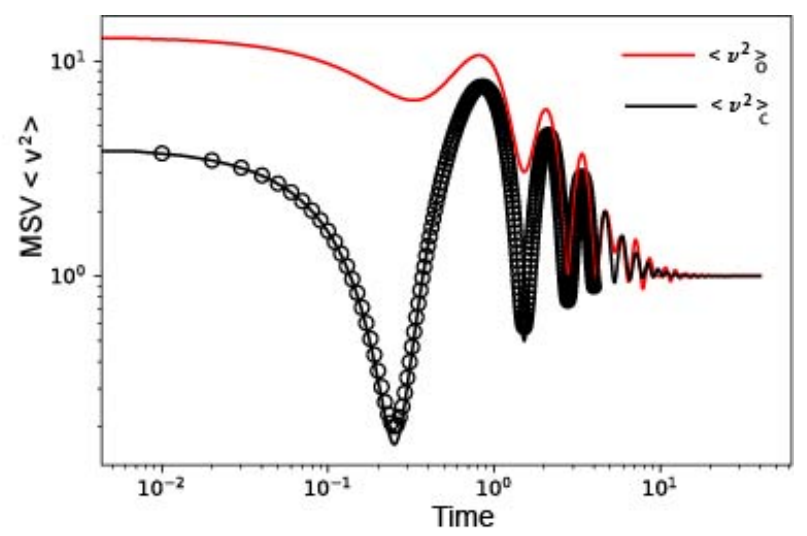

FIGURE 6. Comparison of MSV (54) (red) with both, exact (black) and numerical simulation (circles) results, for values $v_{0}=2$, $x_{0}=1$.

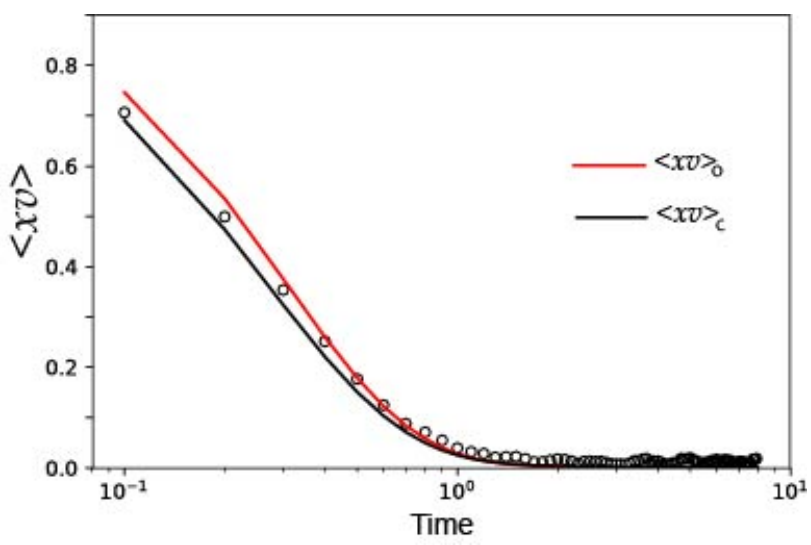

FIGURE 7. CCF (57) (red) compared with exact (black) and numerical simulation (circles) results, for values $v_{0}=1, x_{0}=1$.

$$
\begin{aligned}
\left\langle v^{2}(t)\right\rangle_{C} & =\langle v(t)\rangle^{2}+\frac{k_{B} T}{m}\left\{1-e^{-\beta t}\left[\frac{\beta^{2}}{2 \omega_{1}^{2}} \sin ^{2}\left(\omega_{1} t\right)\right.\right. \\
& \left.\left.-\frac{\beta}{2 \omega_{1}} \sin \left(\omega_{1} t\right)+1\right]\right\}
\end{aligned}
$$

being $\langle v(t)\rangle$ the same as Eq. (55). In the long time limit, both Eqs. (54) and (56) tend to $k_{B} T / m$, as must be. We show in Fig. 6 the comparison between these two results with the numerical simulations. Again, numerical simulations results agree with the exact solution (56) and deviate from the approximate (54). However, the three results coincide for long times.

\subsection{Cross correlation function (CCF)}

Finally, we present the CCF with the Langevin methodology, also in both damped and periodic cases.

\subsubsection{Damped case $(\beta>2 \omega)$.}

The CCF in the damped case is easily obtained from Eq. (47), yielding to

$$
\begin{aligned}
\langle x(t) v(t)\rangle_{O} & =\langle x(t)\rangle\langle v(t)\rangle \\
& +\frac{2 k_{B} T}{m \bar{\beta}_{1}} e^{-\frac{1}{2} \beta t} \sinh \left(\frac{1}{2} \bar{\beta}_{1} t\right) .
\end{aligned}
$$

On the other side, Chandrasekhar's results is given by

$$
\begin{aligned}
\langle x(t) v(t)\rangle_{C} & =\langle x(t)\rangle\langle v(t)\rangle \\
& +\frac{4 \beta k_{B} T}{m \beta_{1}^{2}} e^{-\beta t} \sinh ^{2}\left(\frac{1}{2} \beta_{1} t\right),
\end{aligned}
$$

being $\langle x(t)\rangle$ and $\langle v(t)\rangle$, in both, the same as Eqs. (34) and (51), respectively. We can observe the difference between the second terms of the right hand side of both Eqs. (57) and (58). The comparison with the numerical simulations is shown in Fig. 7. 


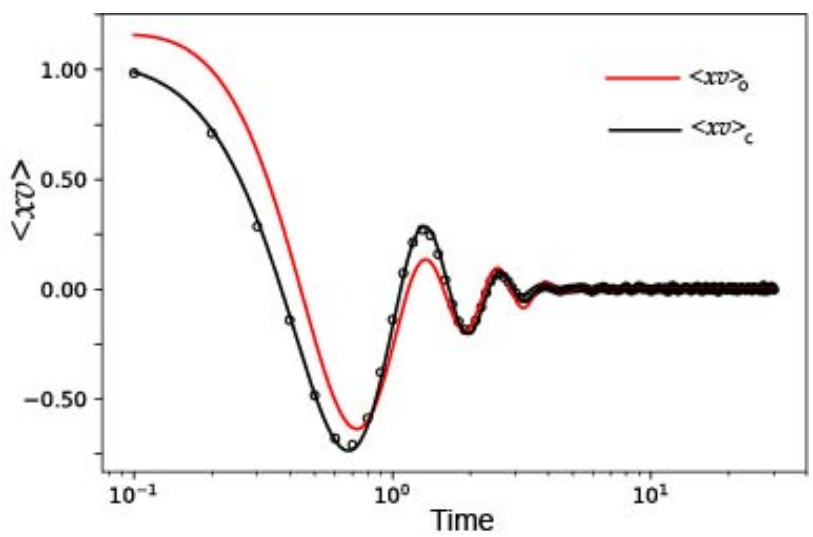

FIGURE 8. Langevin vs. Chandrasekhar COV for damped HOMB with $v_{0}=2, x_{0}=0.5$.

\subsubsection{Periodic case $(\beta<2 \omega)$.}

Here the CCF using Langevin's strategy yields to

$$
\begin{aligned}
\langle x(t) v(t)\rangle_{O} & =\langle x(t)\rangle\langle v(t)\rangle \\
& +\frac{k_{B} T}{\sqrt{2} m \bar{\omega}_{1}} e^{-\beta t} \sin \left(\sqrt{2} \bar{\omega}_{1} t\right) .
\end{aligned}
$$

The CCF reported by Chandrasekhar reads

$$
\langle x(t) v(t)\rangle_{C}=\langle x(t)\rangle\langle v(t)\rangle+\frac{\beta k_{B} T}{m \omega_{1}^{2}} e^{-\beta t} \sin ^{2}\left(\omega_{1} t\right) .
$$

In both expressions for CCF, $\langle x(t)\rangle$ and $\langle v(t)\rangle$, are the same as Eqs. (39) and (55), respectively. In Fig. 8, we report the CCF for the three cases, namely, Langevin's original result (red), exact (black), and numerical simulation (circles) results.

\section{Conclusions}

In this work, Langevin's original strategy to solve the HOBM has been applied. Following the methodology, we have calculated the statistical properties of a HOBM in the damped and periodic cases. In both cases, similar structure are exhibited with certain deviations only in the transient region compared with those reported in $[5,6]$. However, as time increases, our theoretical results are equal to the exact solutions, as it is indeed shown analytically as well as by numerical simulations. Therefore, in the long time limit, Langevin's original strategy is consistent with damped and periodic cases. We emphasize that, the deviations in the transient region are due to the absence of noise at the beginning of the solution of the Langevin equation. This is the reason for the loss of information in the transient regime. Also, the free particle case has been presented, for which unexpected results have been found.

We want to recall that the usual strategy for solving problems related to Brownian motion, relies upon the statistical properties of the noise. However, the Langevin original method, even when it does not provide the exact solution, it is consistent in the long time limit. It is only exact to describe the free particle OU process.

In conclusion, the pedagogical application of Langevin's original strategy in the study of Brownian motion has been barely explored [28]. Therefore, we consider this strategy a useful mathematical tool for graduate courses concerning the theory of Brownian motion.

\section{Appendix}

\section{A. Lemons' results for MSD}

\section{A.1 Periodic case $\gamma<2 \omega$}

The solution for the HOBM proposed by Lemons in chapter 9 of his book relies upon the solution of an Ito-type Langevin equation, in terms of differentials [6]. Using the statistical properties of a Gaussian white noise, Lemons shows that the exact solution for the periodic or lightly damped case $(\gamma<$ $2 \omega)$ for the variance, which we define as $\operatorname{var}\{X\} \equiv \sigma_{x_{L}}^{2}(t)$, is given by

$$
\begin{aligned}
\sigma_{x_{L}}^{2}(t) & =\frac{\beta^{2}}{2 \gamma \omega^{2}}+e^{-\gamma t}\left(\frac{\beta^{2}}{8 \gamma \omega^{\prime 2} \omega^{2}}\right) \\
& \times\left[-4 \omega^{2}+\gamma^{2} \cos 2 \omega^{\prime} t-2 \gamma \omega^{\prime} \sin 2 \omega^{\prime} t\right],
\end{aligned}
$$

where $\omega^{\prime}=\sqrt{\omega^{2}-\left(\gamma^{2} / 4\right)}, \quad \beta$ parameter represents the noise intensity and satisfies the fluctuation-dissipation relation $\beta^{2}=2 \gamma k_{B} T / m, \gamma$ being the friction coefficient. It can be easily shown that Eq. (A.1) can also be written as

$$
\begin{aligned}
\sigma_{x_{L}}^{2}(t) & =\frac{k_{B} T}{m \omega^{2}}\left\{1-e^{-\gamma t}\left(\frac{\gamma^{2}}{2 \omega^{\prime 2}} \sin ^{2} \omega^{\prime} t\right.\right. \\
& \left.\left.+\frac{\gamma}{2 \omega^{\prime}} \sin 2 \omega^{\prime} t+1\right)\right\}
\end{aligned}
$$

and the MSD is

$$
\begin{aligned}
\left\langle x^{2}\right\rangle_{x_{L}} & =\langle x(t)\rangle^{2}+\frac{k_{B} T}{m \omega^{2}}\left\{1-e^{-\gamma t}\right. \\
& \left.\times\left(\frac{\gamma^{2}}{2 \omega^{\prime 2}} \sin ^{2} \omega^{\prime} t+\frac{\gamma}{2 \omega^{\prime}} \sin 2 \omega^{\prime} t+1\right)\right\},
\end{aligned}
$$

with

$$
\begin{aligned}
\langle x(t)\rangle & =x_{0} e^{-\frac{1}{2} \beta t}\left(\cos \omega^{\prime} t+\frac{\beta}{2 \omega^{\prime}} \sin \omega^{\prime} t\right) \\
& +\frac{v_{0}}{\omega^{\prime}} e^{-\frac{1}{2} \beta t} \sin \omega^{\prime} t,
\end{aligned}
$$




\section{A.2 Damped case $\gamma>2 \omega$}

The exact solution for the variance in the damped case $(\gamma>2 \omega)$ has the same algebraic structure as Eq. (A.2), except that the parameter $\omega^{\prime}$ must be replaced by $\beta^{\prime} / 2$, where $\beta^{\prime}=\sqrt{\gamma^{2}-4 \omega^{2}}, \sin \omega^{\prime} t$ and $\sin 2 \omega^{\prime} t$, respectively by $\sinh (1 / 2) \beta^{\prime} t$ and $\sinh \beta^{\prime} t$, yielding to

$$
\begin{aligned}
\sigma_{x_{L}}^{2}(t) & =\frac{k_{B} T}{m \omega^{2}}\left\{1-e^{-\gamma t}\right. \\
& \left.\times\left(\frac{2 \gamma^{2}}{\beta^{\prime 2}} \sinh ^{2} \frac{1}{2} \beta^{\prime} t+\frac{\gamma}{\beta^{\prime}} \sinh \beta^{\prime} t+1\right)\right\},
\end{aligned}
$$

where the MSD

$$
\begin{aligned}
\left\langle x^{2}(t)\right\rangle_{L} & =\langle x(t)\rangle^{2}+\frac{k_{B} T}{m \omega^{2}}\left\{1-e^{-\gamma t}\right. \\
\times & \left.\left(2 \frac{\gamma^{2}}{\beta^{\prime 2}} \sinh ^{2} \frac{1}{2} \beta^{\prime} t+\frac{\gamma}{\beta^{\prime}} \sinh \beta^{\prime} t+1\right)\right\},
\end{aligned}
$$

and

$$
\begin{aligned}
\langle x(t)\rangle & =x_{0} e^{-\frac{1}{2} \gamma t}\left(\cosh \frac{1}{2} \beta^{\prime} t+\frac{\gamma}{\beta^{\prime}} \sinh \frac{1}{2} \beta^{\prime} t\right) \\
& +\frac{2 v_{0}}{\beta^{\prime}} e^{-\frac{1}{2} \gamma t} \sinh \frac{1}{2} \beta^{\prime} t .
\end{aligned}
$$

Equations (A.6) and (A.7) are the same as those reported by Chandrasekhar and given in Eqs. (214) from his 1943 paper.

\section{Acknowledgments}

OCV and NLA thank the support from CONACyT-México. OCV, NLA, and NSS thanks to SIP-IPN (México). The authors also thank M. Díaz for a careful reading of our manuscript.
1. P. Langevin, "Sur la théorie du mouvement brownien", C. $R$. Acad. Sci. 146 (1908) 530; English translation: "On the Theory of Brownian Motion", D. S. Lemons and A. Gythiel Am. J. Phys. 65 (1997) 1079. http://dx.doi.org/10.1119/ 1.18725

2. A. Einstein, Investigations on the theory of the Brownian movement, (Dover Publications, Inc. 1956), Ann. Physik 17 (1905) 549.

3. R. Brown, "On the Particles contained in the Pollen of Plants; and on the General Existence of active Molecules in Organic and Inorganic Bodies", Edinburgh New Philosophical Journal 5 (1828) 358.

4. N. Wax, Selected papers on noise and stochastic processes, (Dover Publications, 1954); W. T. Coffey, Yu. P. Kalmykov, The Langevin Equation: With Applications to Stochastic Problems in Physics, Chemistry and Electrical Engineering, (World Scientific Publishing Company, 2004).

5. S. Chandrasekhar, "Stochastic Problems in Physics and Astronomy", Rev. Mod. Phys. 15 (1943) 1. https://doi.org/ 10.1103/RevModPhys.15.1

6. D. S. Lemons, An Introduction to Stochastic Processes in Physics, (The Johns Hopkins University Press, 2002).

7. D. T. Gillespie, Markov Processes: An Introduction for Physical Scientists, (Elsevier, 1991).

8. R. Czopnik and P. Garbaczewsky, "Brownian motion in a magnetic field", Phys. Rev. E 63 (2001) 021105. https://journals.aps.org/pre/abstract/10. 1103/PhysRevE.63.021105

9. T. P. Simões and R. E. Lagos, "Kramers equation for a charged Brownian particle: The exact solution", Physica A 355 (2005) 274. https://doi.org/10.1016/j. physa.2005.03.034

10. F. N. C. Paraan, M. P. Solon, and J. P. Esguerra, "Brownian motion of a charged particle driven internally by correlated noise",
Phys. Rev. E 77 (2008) 022101. https://doi.org/10. 1103/PhysRevE.77.022101

11. L. J. Hou, Z. L. Miskovic, A. Piel, and P. K. Shukla, "Brownian dynamics of charged particles in a constant magnetic field", Phys. Plasmas 16 (2009) 053705. https: / / doi.org/10. $1063 / 1.3138746$

12. E. Bringuier, "From mechanical motion to Brownian motion, thermodynamics and particle transport theory", Eur. J. Phys. 29 (2008) 1243. https://doi.org/10.1088/ 0143-0807/29/6/013

13. J. Tóthová and G. Vasziová and L. Glod and V. Lisý, "Langevin theory of anomalous Brownian motion made simple", Eur. J. Phys. 32 (2011) 645. https://doi.org/10.1088/ $0143-0807 / 32 / 6 /$ L04

14. D. S. Lemons and D. L. Kaufman, "Brownian Motion of a Charged Particle in a Magnetic Field", IEEE Transactions of Plasma Science, 27 (1999) 1288.

15. E. Bringuier, "On the Langevin approach to particle transport", Eur. J. Phys. 27 (2006) 373. https : / / doi .org/10. 1088/0143-0807/27/2/019

16. J. I. Jiménez-Aquino and M. R. Romero-Bastida, "Brownian motion of a charged particle in a magnetic field", Rev. Mex. Fis. E 52 (2006) 182.

17. J. I. Jiménez-Aquino, and R. M. Velasco, and F. J. Uribe, Brownian motion of a classical harmonic oscillator in a magnetic field, Phys. Rev. E 77 (2008) 051105. https://doi.org/ $10.1103 /$ PhysRevE.77.051105

18. M.Yaghoubi, M. E. Foulaadvand, A. Bérut and J. Luczka, Energetics of a driven Brownian harmonic oscillator, J. of Stat. Mech. Theo. and Exp. 2017 (2017) 113206.

19. M. Mandrysz and B. Dybiec, "Energetics of the undamped stochastic harmonic oscillator", A. Phy. Pol. B 49 (2018) 871. 
20. S. F.N $\phi$ rrelykke and H. Flyvbjerg, "Harmonic oscillator in heat bath: Exact simulation of time-lapse-recorded data and exact analytical benchmark statistics", Phys. Rev. E 83 (2011) 041103. https://doi.org/10.1103/PhysRevE.83. 041103

21. M. J. Madsen and A. D. Skowronski, "Brownian motion of a trapped microsphere ions", Am. J. Phys. 82 (2014) 934. https://doi.org/10.1119/1.4881609

22. G. Volpe, "Simulation of a Brownian particle in an optical trap", Am. J. Phys. 81 (2013) 224. https : / / doi .org/10 . $1119 / 1.4772632$

23. T. Li and M. G. Raizen, "Brownian motion at short time scales", Ann. Der Phys. 525 (2013) 281. https://doi.org/10. 1002 /andp.201200232

24. A. Darras, J. Fiscina, N. Vandewalle, and G. Lumay, "Relating Brownian motion to diffusion with superparamagnetic col- loids", Am. J. Phys. 85 (2017) 265. https://doi.org/ $10.1119 / 1.4975382$

25. Y. Pomeaua and J. Piasecki, "The Langevin equation", C. R. PHYS. 18 (2017) 570. https://doi.org/10.1016/j. crhy.2017.10.001

26. R. Newburgh, J. Peidle, and W. Rueckner "Einstein, Perrin, and the reality of atoms: 1905 revisited", Am. J. Phys. 74 (2006) 478. https://doi.org/10.1119/1.2188962

27. K. Burrage, I. Lenane and G. Lythe, Numerical Methods for Second-Order Stochastic Differential Equations, Siam J. Sci. Comput. 29 (2007) 245. https://doi.org/10.1137/ 050646032

28. N. Lucero-Azuara, N. Sánchez-Salas, and J.I. Jiménez-Aquino "Brownian motion across a magnetic field: Langevin approach revisited". Eur. J. Phys. 41 (2020) 3. https://doi.org/ $10.1088 / 1361-6404 / \mathrm{ab} 6 \mathrm{c} 2 \mathrm{~b}$ 